\title{
HOLLINÉLLIDOS DEL MOSCOVIENSE SUPERIOR (CARBONÍFERO) DEL ESTE DE ASTURIAS (N DE ESPAÑA)
}

\author{
Luis C. SÁNCHEZ DE POSADA' y Martin J. M. BLESS \\ ' Departamento de Geología. Universidad. Oviedo. España \\ 'Looierstraat 3. 6271 BA Gulpen. Holanda
}

Sánchez de Posada, L.C. y Bless, M.J.M. 1999. Hollinéllidos del Moscoviense Superior (Carbonífero) del este de Asturias ( $\mathrm{N}$ de España).[Upper Moscovian (Carboniferous) hollinellids from eastern Asturias (N Spain)]. Revista Española de Paleontología, $\mathbf{n}^{\mathbf{0}}$ extr. Homenaje al Prof. J. Truyols, 29-41. ISSN 0213-6937.

\begin{abstract}
An about 1,500 $\mathrm{m}$ thick succession (Playa de la Huelga section) of mainly limestones, ranging in age from Tournaisian to Late Moscovian ("Myachkovsky"), is exposed in cliffs along the coast near the small village of Hontoria (Llanes, Asturias, N Spain). Due to its good exposure, the long stratigraphical interval and the type of facies, this section deserves special attention. Several species of foraminifers, brachiopods, corals, bivalves (more abundant in the upper part of the section) have been described up to now from this succession.

Etching several kilogrammes of limestones by means of formic and acetic acids provided a very prolific silicified fauna of ostracodes (along with other fossils) consisting of more than 50 species (mainly belonging to the genera Kirkbya, Coronakirkbya, Amphissites, Roundyella, Bairdia, Bohlenatia, Acratia, Acantoscapha and Bairdiacypris) which are now being studied by the first author. A remarkable part of this fauna is the genus Hollinella Coryell, 1928, which is represented by three different species. One of them (previously known from Bashkirian rocks of the Cantabrian Zone) is closely related to Hollinella (Hollinella) bassleri (Knight, 1928), an ostracode quite common in the Pennsylvanian strata of the USA. Another species (described as Hollinella $(H$. llanisca n. sp. in this paper) is probably related to Hollinella (H.) cristinae Bless, 1965 and Hollinella (H.) dentata Coryell, 1928. The third species is characterized by a peculiar configuration of the adventral structures hirherto unknown in the genus. Consequently the latter is assigned to a new subgenus of Hollinella: Hollinella (Truyolsella) n. subgen.

On the whole the three species described herein show remarkable similarities with the species of Hollinella described by Fohrer from the Gzhelian of the Carnic Alps.
\end{abstract}

Keywords: Ostracodes, Hollinella, systematics, Carboniferous, Upper Moscovian, Cantabrian Zone, Asturias, Spain.

\section{RESUMEN}

En las proximidades de Hontoria (Llanes, Asturias) aflora una potente sucesión (de aproximadamente $1.500 \mathrm{~m}$ de potencia) en facies casi exclusivamente calcárea, que incluye materiales cuya edad está comprendida entre el Turnesiense y el Moscoviense Superior (tramos equivalentes al Horizonte de Myachkovo) y que usualmente es conocida como sección de la Playa de la Huelga. Por su exposición, espesor de materiales propios de régimen de plataforma carbonatada y abundancia de restos fósiles en algunos niveles, esta sucesión merece atención especial. En diversos tramos se han descrito restos pertenecientes a distintos grupos fósiles: foraminíferos, corales, braquiópodos y bivalvos, que, en conjunto, son más abundantes en la parte alta.

La disolución mediante ácidos fórmico y acético de varios kilogramos de caliza permitió obtener una variadísima fauna de ostrácodos, que está siendo estudiada por el primer autor y que probablemente está integrada por más de una cincuentena de especies pertenecientes, entre otros, a los géneros Kirkbya, Coronakirkbya, Amphissites, Roundyella, Bairdia, Bohlenatia, Acratia, Acantoscapha y Bairdiacypris. Entre ellas destacan tres especies del género Hollinella Coryell, 1928. Una, conocida ya en niveles más bajos de la propia Zona Cantábrica, está relacionada con Hollinella (Hollinella) bassleri (Knight, 1928), ampliamente representada en el Carbonífero de los Estados Unidos de N América; otra, descrita aquí como Hollinella $(H$. ) llanisca n. sp., parece estar relacionada con Hollinella (H.) cristinae Bless, 1965 y Hollinella ( $H_{\text {.) }}$ dentata Coryell, 1928 y, finalmente, la tercera, Hollinella (Truyolsella) truyolsi n. subgen., n. sp., presenta una 
configuración de las estructuras adventrales desconocida hasta el momento en el género, lo que nos induce a considerarla como especie tipo de un nuevo subgénero.

En conjunto, las tres especies descritas en este trabajo muestran considerables semejanzas con las especies de Hollinella descritas por Fohrer en el Gzheliense de los Alpes Cárnicos.

\section{Palabras clave: Ostrácodos, Hollinella, sistemática, Carbonífero, Moscoviense Superior, Zona Cantábrica, Asturias, España.}

\section{INTRODUCCIÓN}

La familia Hollinellidae constituye uno de los grupos más relevantes del orden Palaeocopida. Durante el Carbonífero Superior está representada esencialmente por tres géneros: Hollinella Coryell, 1928, Jordanites Bless, 1967 y Gortanella Ruggieri, 1966. El primero de ellos, el más abundante, aparece en materiales depositados en numerosos ambientes marinos, a veces formando una fracción importante de las asociaciones.

Contrastando con lo que sucede en el Carbonífero Superior de otras regiones del mundo, fundamentalmente en los Estados Unidos de $\mathrm{N}$ América, el número de especies de Hollinella descritas hasta ahora en Europa Occidental no es grande. Varias de las formas conocidas en el NO de Europa -Hollinella $(H$.) spinulosa Demanet, 1949, H. (H.) ulrichi (Knight, 1928), H. (Praehollinella) sentinellensis Bless y Jordan, 1972- fueron halladas en la banda marina del Aegir y equivalentes (que constituyen la base del Bolsoviense, ex Westfaliense C); Hollinella (P.) claycrossensis Bless y Calver, 1970 se encontró en el Duckmantiense, ex Westfaliense B y en la base del Bolsoviense y Hollinella $(H$.) cristinae Bless, 1965 se conoce ya en el Langsettiense (ex Westfaliense A). Por su parte, Fohrer (1991) dio a conocer la presencia de Hollinella $(H$.$) ulrichi (Knight, 1928) en el Gzheliense$ (Estefaniense) de los Alpes Cárnicos. El resto de los hallazgos de representantes del género, Hollinella $(H$. micheli Bless, 1968, $H$. (H.) philomenae Bless, 1967, $H$. (P.) camoni Bless, $1968, H$. (P.) sp. aff. camoni Bless, 1968, H. (H.) bassleri (Knight, 1928), H. (H.) aff. bassleri (Knight, 1928), H. (Keslingella) cf. radiata (Jones y Kirkby, 1886), $H$. (H.) sp. gr. dentata Coryell, 1928, Hollinella (H.) sp. A Becker, 1978, Hollinella? cf. avonensis (Latham, 1932), procede de la Cordillera Cantábrica (v. Bless, 1965, 1967, 1968, 1969, Sánchez de Posada, 1977, Becker, 1978, Fernández López y Sánchez de Posada, 1987), de donde, además, fue descrita originalmente Hollinella ( $H$.) cristinae.

No es sorprendente que el mayor número de formas haya sido encontrado en la Zona Cantábrica, toda vez que esta región contiene las únicas sucesiones dentro del Carbonífero Superior de Europa Occidental con carácter marino o predominantemente marino durante amplios intervalos estratigráficos.

En este trabajo se describen tres especies de Hollinella halladas en forma de valvas silicificadas en calizas dentro de una de las secciones (la de la Playa de la Huelga) más completas del Carbonífero marino de la Zona Cantábrica. De este modo, las formas aquí descritas complementan los datos que se tienen de las asociaciones faunísticas presentes en una sucesión que, como se expone a continuación, tiene un carácter muy especial dentro del contexto de Europa Occidental.

La fauna de ostrácodos de Hontoria, la de los Alpes Cárnicos, descrita por Fohrer (1991) y la de las Bükk Mountains estudiada por Kozur (1985) representan las asociaciones más ricas y variadas de ostrácodos encontradas en calizas de esta edad a escala mundial. En este sentido, pueden ser consideradas como tres importantes puntos de referencia para el conocimiento de la composición de las asociaciones de ostrácodos que poblaron las plataformas carbonatadas en los tiempos finales del Carbonífero.

\section{MARCO GEOLÓGICO}

La Zona Cantábrica es la más externa de las unidades en que Lotze (1945) dividió el Macizo Ibérico. En ella están expuestos materiales que abarcan desde el Precámbrico hasta el Pérmico, ocultos, en algunos puntos, bajo una cobertera postpaleozoica. Dentro de la Zona Cantábrica se distinguen usualmente varias unidades (Somiedo-Correcillas, Sobia-Bodón, Aramo, Cuenca Carbonífera Central de Asturias, Ponga, Picos de Europa y Unidad Palentina) establecidas con criterios estructurales y estratigráficos por Julivert (1971) y modificadas ligeramente por Pérez-Estaún et al. (1988). Cada una de estas unidades representa un manto compuesto a su vez por varias escamas plegadas, dispuestas de modo que las más occidentales (o las más septentrionales) cabalgan sucesivamente a las más orientales (o a las más meridionales) (Fig. 1A).

Los materiales Carboníferos afloran en todas las unidades que integran la Zona Cantábrica, aunque su naturaleza es muy diferente. Dentro de la Unidad de Picos de Europa y en la rama $\mathrm{N}$ de las escamas más externas (situadas hacia el interior del arco que dibujan las estructuras) de la Unidad del Ponga, el Carbonífero está representado por sucesiones calcáreas o casi totalmente calcáreas que reemplazan la alternancia de materiales detríticos y carbonáticos existentes en otros lugares (v. Sánchez de Posada et al., 1990, 1996 para una descripción sintética del desarrollo del Carbonífero marino cantábrico).

\section{LA SECCIÓN DE LA PLAYA DE LA HUELGA}

La sección de la Playa de la Huelga se localiza en la costa E de Asturias, unos $10 \mathrm{Km}$ al E de Llanes, entre la Playa de San Antolín (Km 107 de la carretera Nacional 


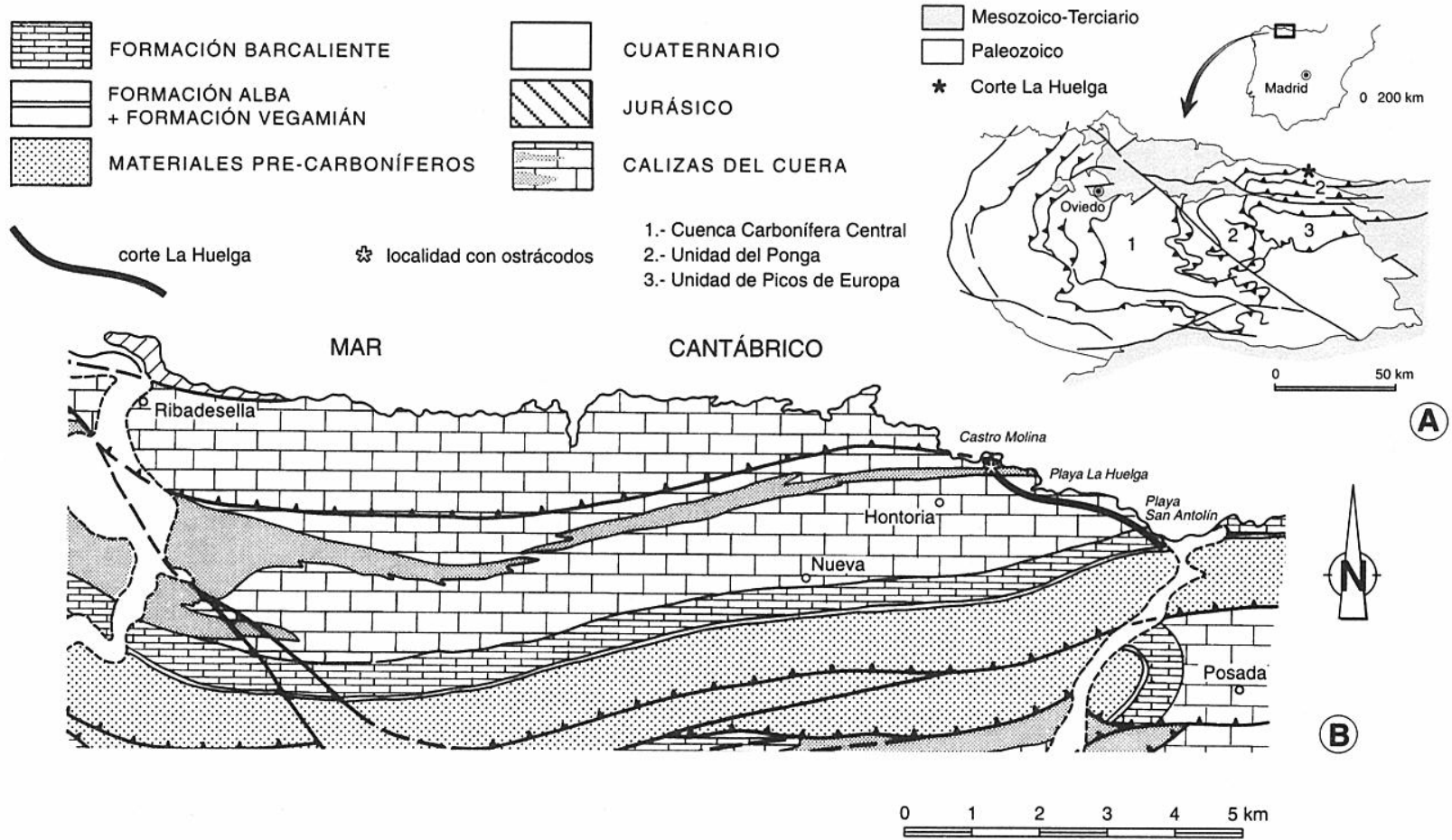

Figura 1. A Esquema general de la Zona Cantábrica en el que se muestra la localización de la sección de la playa de la Huelga dentro de la Unidad del Ponga y la situación de esta última respecto a las unidades contiguas (Cuenca Carbonífera Central de Asturias y Unidad de Picos de Europa). B. Esquema geológico regional con la situación de la sección de la playa de la Huelga.

634) y Castro Molina, unos $850 \mathrm{~m}$ al $\mathrm{N}$ del pequeño pueblo de Hontoria (Fig. 1B).

La investigación llevada a cabo en los últimos años por diversos autores permite afirmar que en dicha sucesión se encuentra representada, con facies casi totalmente calcárea, una parte importante del Carbonífero (desde al menos el Turnesiense hasta el Moscoviense Superior). De este modo, dicha sección (como las expuestas en algunos enclaves de la región de Picos de Europa) tiene una naturaleza poco frecuente dentro del Carbonífero del mundo y, por ello, merece especial atención.

Los materiales carboníferos (Fig. 2) se apoyan sobre cuarcitas y areniscas del Ordovícico y Devónico Superior. Un importante hiato determina la ausencia de materiales silúricos y devónicos previos al Fameniense. Los 20 primeros metros (expuestos parcialmente) están formados por un tramo condensado de edad comprendida entre el Turnesiense y el Arnsbergiense, constituido por liditas negras (Fm. Vegamián, esencialmente Turnesiense) y calizas rojas nodulosas con intercala-ciones de pizarras (Formación Alba, Genicera o "Caliza Griotte”).

Por encima se desarrolla una serie calcárea de unos $1.400 \mathrm{~m}$ de potencia, solo interrumpida por tres pequeñas intercalaciones siliciclásticas contenidas en los últimos $400 \mathrm{~m}$ de sucesión. Los primeros $250 \mathrm{~m}$ corresponden a la Fm. Barcaliente (calizas negras, tableadas, pobres en fósiles) presente en una gran extensión de la Zona Cantábrica. El resto está integrado por calizas grises, casi siempre claras, de diferentes tipos, que han sido denominadas por Navarro et al. (1986) "Calizas del
Cuera" y que equivalen a lo que en otros enclaves constituye el conjunto de las formaciones Valdeteja y Picos de Europa.

Los primeros datos paleontológicos de esta sección se deben a Barrois (1882) quien describió varias especies de foraminíferos, corales, braquiópodos, briozoos y bivalvos dentro de lo que él consideró que formaba parte de su "Assise de Lena". Salvo por referencias ocasionales, la sección permaneció largo tiempo ignorada hasta que Martínez Chacón en un conjunto de artículos (Martínez Chacón, 1975, 1977, 1979, 1990, 1991) describió 45 especies de braquiópodos encontrados casi en los mismos niveles que los ostrácodos descritos en este trabajo. Varios trabajos de Villa (esencialmente Villa, 1985, 1995) dedicados al estudio de los foraminíferos, junto con el de Navarro et al. (1986) proporcionaron valiosos datos estratigráficos de estos materiales, estableciendo de modo preciso sus edades en términos de la escala estratigráfica de la antigua Unión Soviética. Finalmente, Rodríguez, 1984, y Rodríguez y Ramírez (1987) describieron diez especies de rugosos y tres de siringopóridos (tabulados) de esta misma localidad.

Los ostrácodos estudiados en este trabajo proceden de la parte alta de la sucesión (Fig. 2), de tramos muy próximos al nivel B-127 de Martínez Chacón (1979, 1988, 1990), que corresponden a niveles considerados por Villa (1985) como Moscoviense Superior (equivalentes a la parte alta del horizonte the Podolsk o a la parte baja del Horizonte de Myachkovo). 


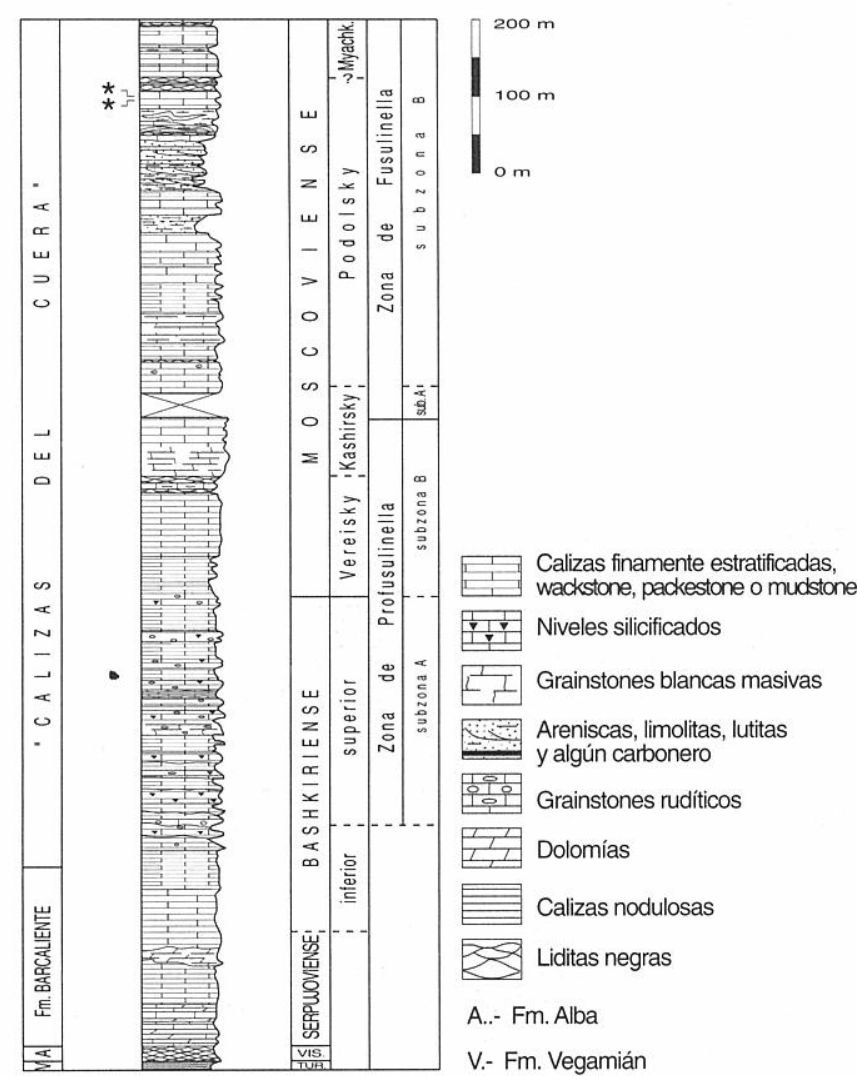

Figura 2. Columna estratigráfica de los materiales carboníferos de la sección de la playa de la Huelga con la posición de las muestras de las que fueron extraidos los ostrácodos estudiados en este trabajo (muestra inferior B-260, muestra superior B-260a).

\section{SISTEMÁTICA}

SUBCLASE OSTRACODA Letreille, 1802

ORDEN PALAEOCOPIDA Henningsmoen, 1953

SUBORDEN HOLLINOMORPHA Henningsmoen, 1965

Superfamilia HOLLINACEA Swartz, 1936

Familia Hollinellidae Bless y Jordan, 1971

Género Hollinella Coryell, 1928

Subgénero Hollinella (Truyolsella) n. subgen.

Especie tipo: Hollinella (Truyolsella) truyolsi n. sp.

Derivatio nominis: El nuevo subgénero se nombra en honor del Prof. Truyols, Departamento de Geología de la Universidad de Oviedo, en reconocimiento a su importante contribución al desarrollo de la Paleontología española.

\section{Diagnosis}

Subgénero de Hollinella en el que la porción anteroventral del velum de uno de los dimorfos adultos está fuertemente incurvada en dirección sagital, formando una bolsa totalmente cerrada, netamente separada de la región posterior del velum por un brusco cambio en la dirección de éste. Valvas pertenecientes a los estadios juveniles provistas de dos espolones semejantes a los de
Hollinella (Keslingella) Bless y Jordan, 1970 y Gortanella Ruggieri, 1966.

One of the dimorphic adults with anteroventral part of the velum inflated, strongly incurved inwards forming a closed cavity on the inner side of the valve, sharply set off from the posterior part of the velum by an abrupt change in the direction of the velum, the posterior part being not incurved. Juvenile instars provided with two adventral spurs like those in Hollinella (Keslingella) Bless \& Jordan, 1970 and Gortanella Ruggieri, 1966.

\section{Discusión}

El contorno general del caparazón y la lobación concuerdan con los típicos de Hollinella. Lo mismo ocurre con la configuración del velum en las formas adultas, provistas de velum alabeado y con las estructuras adventrales de los ejemplares juveniles. En estos últimos son bien patentes dos pequeños espolones iguales a los propios de Hollinella (Keslingella).

Las características más distintivas del nuevo subgénero ( $y$, en consecuencia, también de la nueva especie) se encuentran, a nuestro entender, en las estructuras adventrales de las formas adultas, con velum fuertemente incurvado y se manifiestan fundamentalmente en la formación de una bolsa totalmente cerrada en posición anteroventral, netamente separada de la región más posterior del velum por un brusco cambio en la dirección de éste (v. Fig. 3A, D).

Otras especies de Hollinella tienen también velum fuertemente incurvado, delimitando un espacio cerrado o casi cerrado (como sucede, por ejemplo, en Hollinella (Hollinella) bassleri, pero los límites de este espacio son mucho menos claros, tanto interna como externamente (las diferencias entre ambas constituciones pueden observarse comparando las figuras $6 \mathrm{~A}$ con $3 \mathrm{~A}$ y $6 \mathrm{D}, \mathrm{E}$ con 3D). Todo ello determina la clara división del velum en segmentos: un segmento anterior a la incurvación anteroventral, otro correspondiente a dicha incurvación y un tercero representado por la porción posterior a ésta, de la que está netamente separado.

Al menos en Hollinella (Truyolsella) truyolsi la división mencionada está atestiguada también por importantes diferencias ornamentales: la región hinchada tiene varias filas de papilas, mientras que la región posterior a ella no es papilosa o las papilas están muy reducidas. Este hecho contrasta con la continuidad de la ornamentación del velum en otras especies de Hollinella con velum incurvado, pertenecientes a otros subgéneros, tal como sucede en Hollinella (Hollinella) aff. bassleri (v. Fig. 6D, E).

\section{Hollinella (Truyolsella) truyolsi n. sp. Figs. 3, 4}

Derivatio nominis: La nueva especie se nombra en honor del Prof. Truyols, Departamento de Geología de la Universidad de Oviedo, en reconocimiento a su sincera amistad y permanente magisterio. 

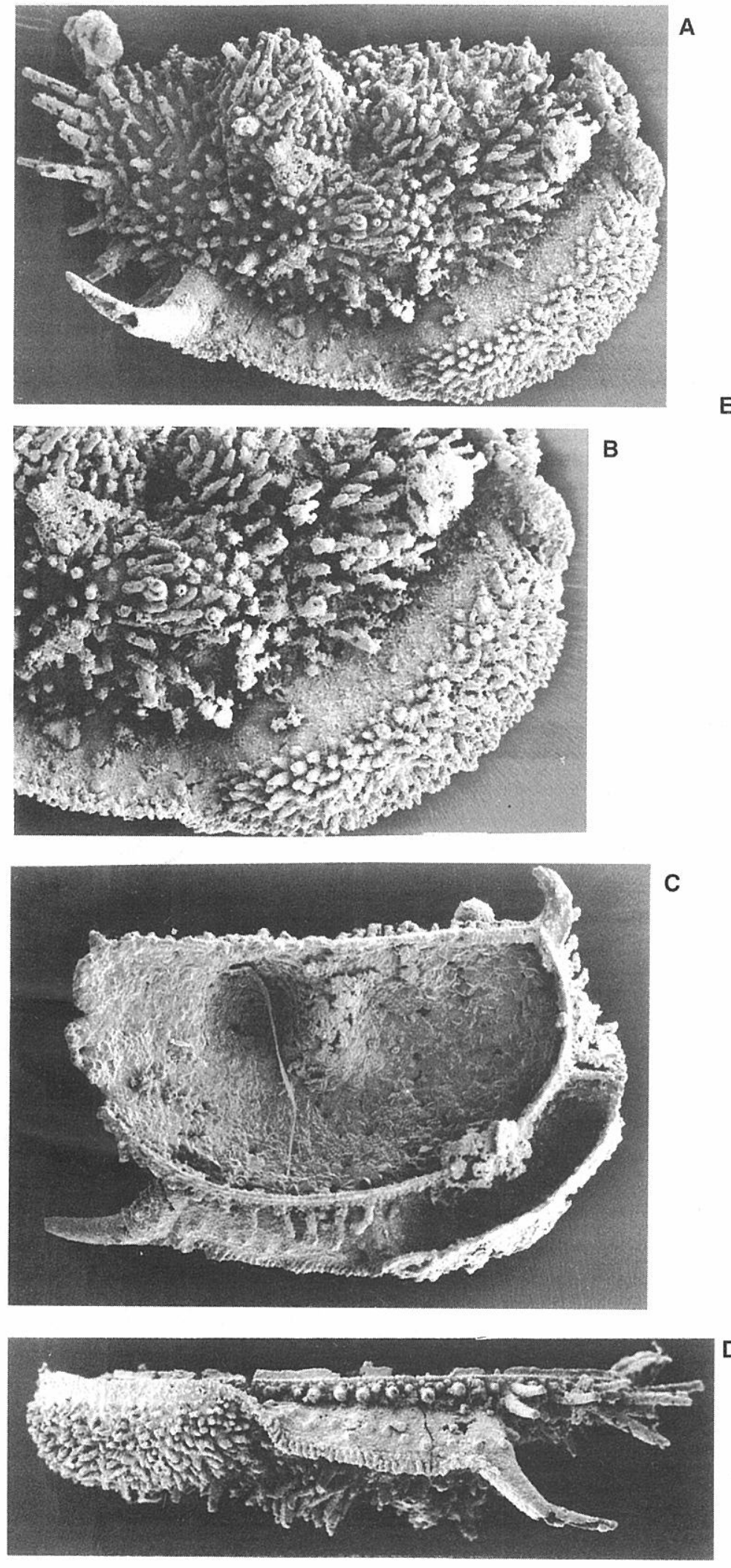
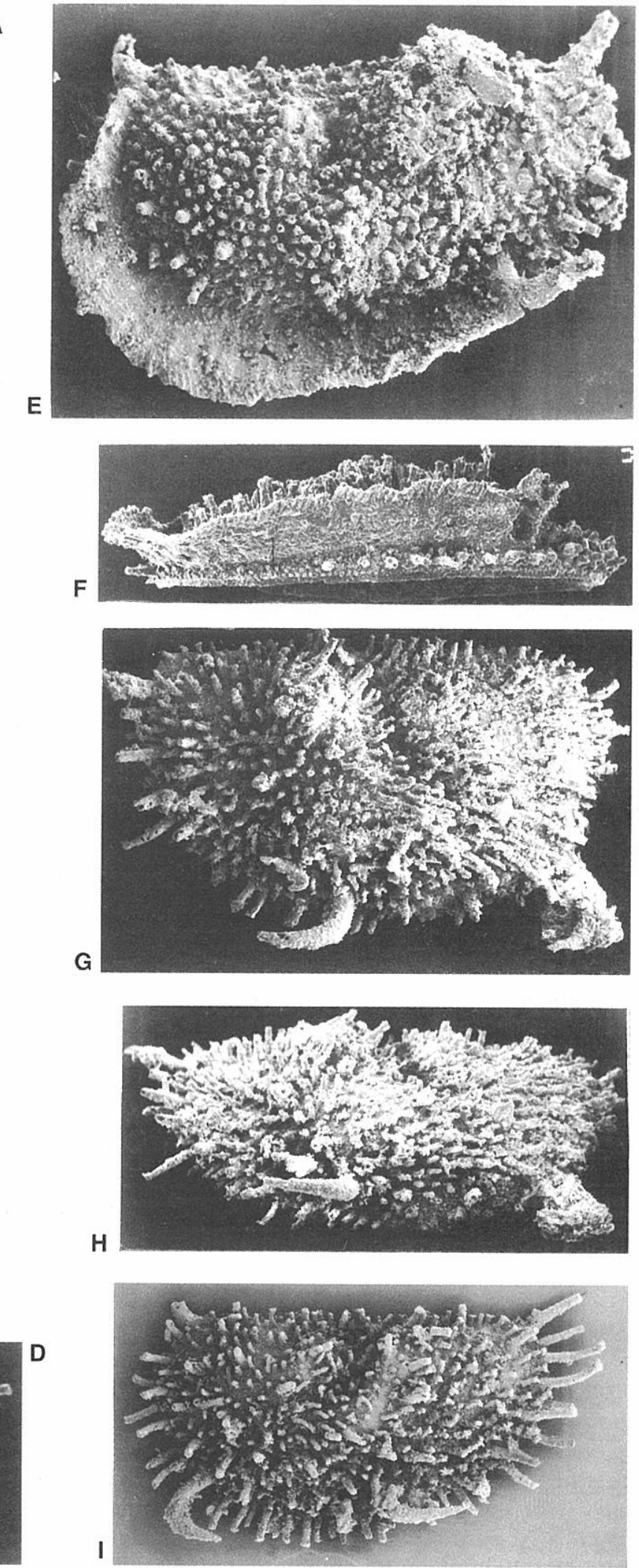

Figura 3. Hollinella (Truyolsella) truyolsi n. subgen., n. sp. A-D. Vistas lateral, detalle de la región anteroventral, vista interna y ventral de valva derecha (Holotipo DPO 32.461). E, F. Vistas lateral y ventral de valva izquierda (Paratipo DPO 32.467). G, H. Vista lateral y vista inclinada unos 45 grados de valva izquierda mostrando la inserción de los espolones adventrales (Paratipo 32.475). I. Vista lateral de valva izquierda (Paratipo DPO 32.473). Las figuras A-D corresponden a un ejemplar adulto con velum fuertemente incurvado. Las figs. E, F, a un ejemplar adulto con velum no incurvado en dirección sagital y las figs. G-I, a ejemplares juveniles. Todas las figuras aproximadamente x65, excepto la B (aproximadamente $\mathrm{x} 75$ ). 


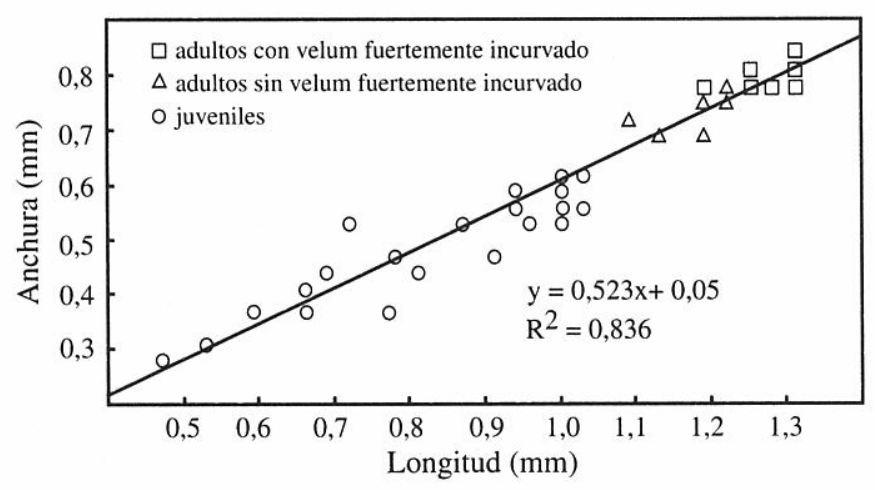

Figura 4. Diagrama L/H de 38 ejemplares de Hollinella (Truyolsella) truyolsi $\mathrm{n}$. $\mathrm{sp}$.

\section{Material}

Holotipo: una valva derecha de ejemplar adulto con velum fuertemente incurvado DPO 32.461 (Figs. 3A-D). Paratipos: 14 valvas, izquierdas y derechas, de ejemplares adultos con velum fuertemente incurvado (DPO 32.459, DPO 32.460, DPO 32.462-DPO 32.466), 10 valvas, izquierdas y derechas, de ejemplares adultos sin fuerte incurvación en el velum (DPO 32.467-32.473, 32531), 25 valvas, izquierdas y derechas, de ejemplares juveniles (DPO 32.473-32.480).

Además se han obtenido unas 30 valvas (DPO 32.481) de ejemplares juveniles de Hollinella, que probablemente pertenezcan a esta especie, pero que no conservan ninguno de los espolones adventrales característicos de los estadios preadultos de esta especie.

Todo el material procede de la muestra B-260a.

Localidad típica: Sección de La Huelga, aproximidamente $850 \mathrm{~m}$ al $\mathrm{N}$ del pueblo de Hontoria (Llanes, Asturias).

Estrato tipico: "Calizas del Cuera". Calizas fuertemente silicificadas con abundante fauna coralina en los tramos altos de la sección de Hontoria, unos $15 \mathrm{~m}$ por encima de la última intercalación siliciclástica. Muestra B-260a (Fig. 2). Moscoviense Superior (tramos equivalentes a la parte alta del horizonte de Podolsk o a la parte baja del horizonte de Myachkovo).

\section{Diagnosis}

Especie de Hollinella (Truyolsella) de tamaño medio, con la superficie preaductorial casi plana, L2 muy pequeño y $\sin$ L1. Superficie lateral granulosa, con numerosas espinas. Tiene espinas cardinales y espinas marginales posteriores bastante largas. Parte hinchada del velum de uno de los dimorfos provista de numerosas papilas en su porción distal (en sentido radial), porción proximal lisa.

A species of Hollinella (Truyolsella) with almost flat preaductorial area, L2 being very small and $L 1$ inconspicuous. Lateral surface granulose, with abundant spines. Anterodorsal and posterodorsal corners provided with cardinal spines. Posterior marginal spines quite long. Distal part of the inflated portion of the velum with closely packed papillae, proximal part smooth.

\section{Descripción}

Ejemplares adultos: Caparazón de tamaño medio, con contorno lateral algo prepleto. Borde dorsal recto, borde ventral convexo. Bordes anterior y posterior redondeados, el anterior formando un arco mucho más largo que el posterior. Superficie preaductorial casi plana, con L2 muy pequeño, casi inexistente y superficie uniformemente convexa por delante de L2, sin L1. L3 alto, hemisférico, rebasando levemente el borde dorsal. L2 y L3 conectados ventralmente por un lóbulo ventral de tamaño moderado. El surco medio se extiende desde casi el borde dorsal hasta el lóbulo ventral. Ángulos cardinales provistos de dos espinas que no siempre se conservan. La anterior tiene forma de gancho, está dirigida en sentido dorsal y doblada hacia atrás; la trasera está dirigida en sentido dorsoposterior. Superficie lateral granulosa con numerosas espinas. Ángulo cardinal anterior obtuso, posterior casi recto. Espinas marginales bastante largas.

Dimorfismo sexual muy aparente. Ambos sexos poseen velum ancho e incurvado, que se extiende desde el ángulo cardinal anterior hasta aproximadamente el límite entre las regiones ventral y posterior, y que termina en un fuerte espolón.

En uno de los sexos, el velum está alabeado y, en conjunto, claramente inflexionado en sentido lateral. La porción más distal de la región anteroventral está algo incurvada en dirección sagital y la parte anterior del velum llega a ser casi perpendicular al plano sagital. La estructura radial del velum es bastante patente.

En el otro sexo, el velum es netamente distinto, sobre todo en la región anteroventral, en la que llega a constituir una bolsa claramente delimitada del resto del velum, tanto anterior como posteriormente y visible tanto desde el exterior como desde el interior del caparazón. En posición anterior respecto al hinchamiento anteroventral, el velum está constituido por una cresta poco expandida, separada del mencionado hinchamiento por una clara constricción. En la región situada por detrás del hinchamiento, el velum es bastante ancho (en sentido radial), oblicuo respecto al plano sagital y termina en un fuerte espolón curvado, bien delimitado, que se inserta en el domicilium. La parte hinchada del velum está provista en su región distal (en sentido radial) de numerosas papilas; su parte proximal es lisa. La región posterior del velum tiene marcada estructura radial y carece de papilas. Por detrás del velum existen espinas marginales bastante largas. La superficie subvelar está también provista de espinas.

Ejemplares juveniles: Su contorno lateral es bastante similar al de los adultos. La lobación está mucho menos desarrollada. El surco medio no es muy marcado. L2 y L3 son muy tenues. La superficie lateral es fuertemente espinosa.

Las estructuras adventrales están formadas por dos espolones bien desarrollados y curvados hacia atrás. El posterior se sitúa en posición ventroposterior y el anterior en el límite entre los bordes anterior y ventral. En algunos ejemplares no están conservados y en los primeros estadios encontrados no existen, aunque ello es casi con seguridad efecto de la conservación. A lo largo de los bordes libres aparece un conjunto de numerosas espinas, bastante largas y 

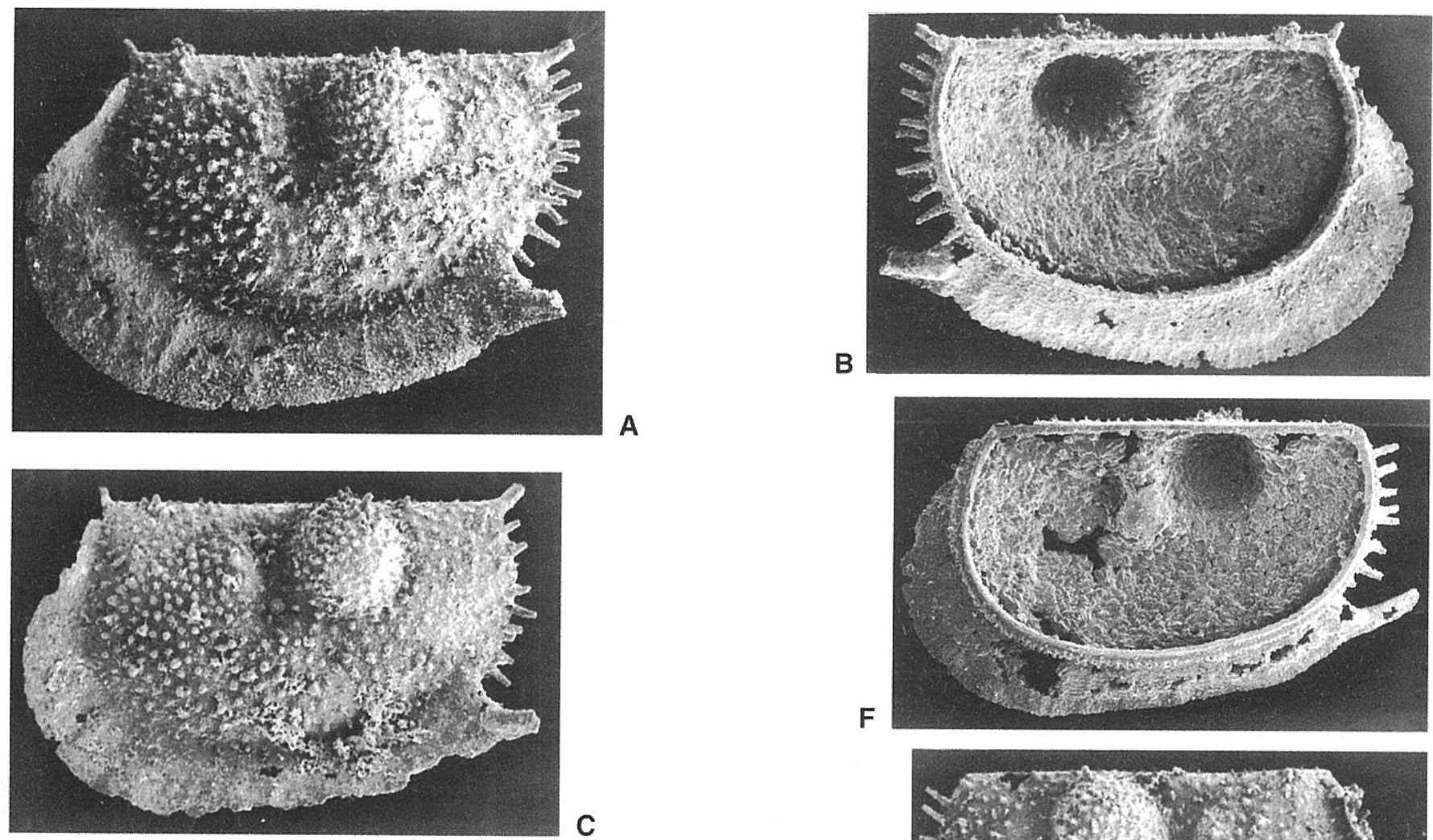

\section{A}
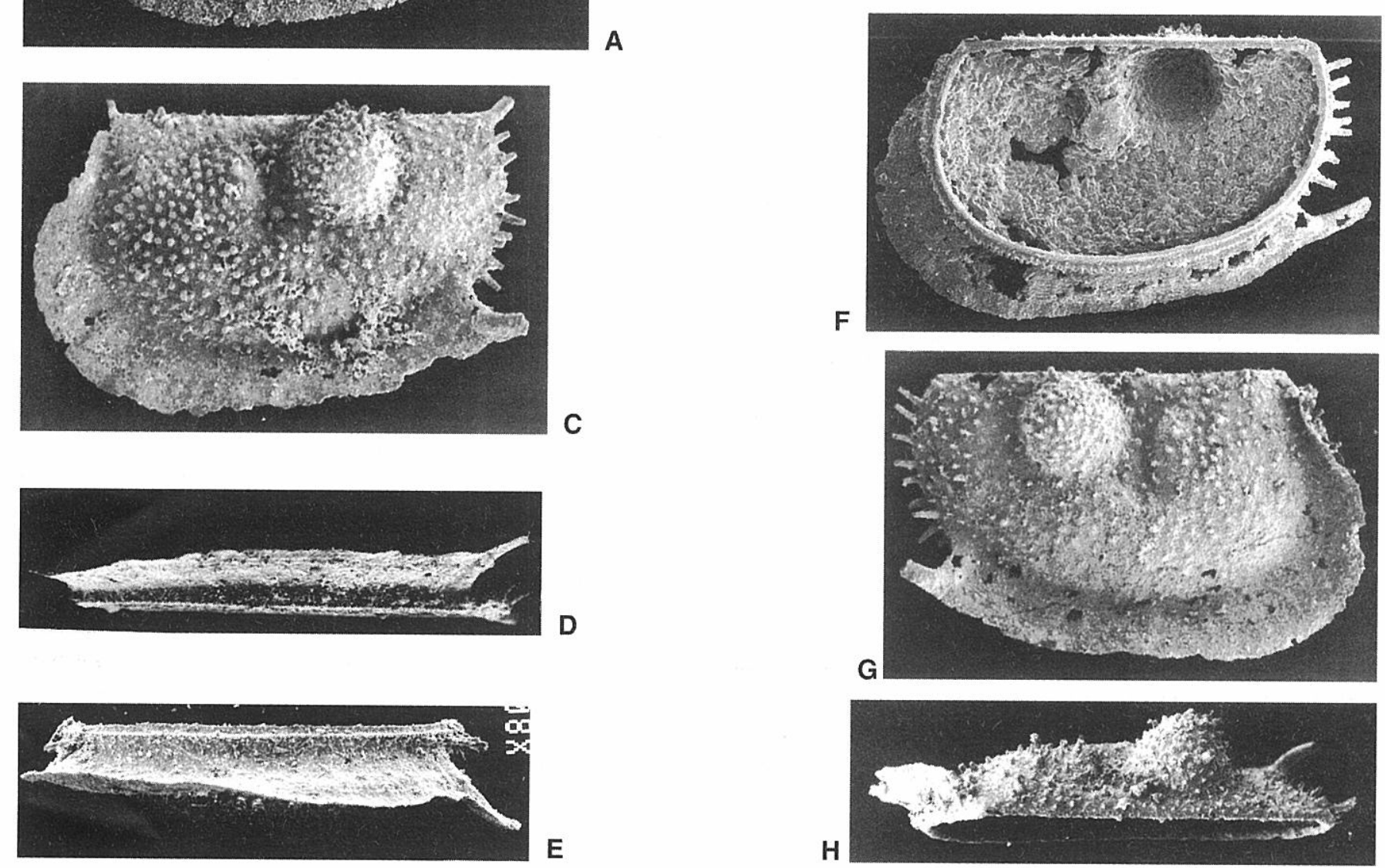

E

H

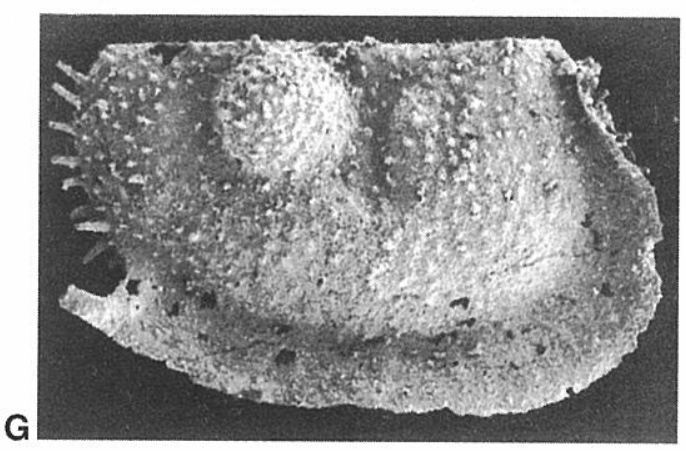

Figura 5. Hollinella (Hollinella) llanisca n. sp. A, B. Vistas lateral externa e interna de una valva izquierda (Holotipo DPO 32.482). C, D. Vistas lateral y ventral de valva izquierda (Paratipo DPO 32.483). E. Vista ventral de una valva derecha (Paratipo DPO 32.494). F. Vista lateral interna de una valva izquierda (Paratipo DPO 32.493). G, H. Vistas lateral y dorsal de una valva derecha (Paratipo DPO 32.485). Todas las figuras representan ejemplares adultos. Las figuras A, B, E y F representan ejemplares con velum incurvado en dirección sagital, el resto, valvas con velum no incurvado. Todas las figuras aproximadamente $\mathrm{x} 70$.

dispuestas en varias hileras. El carácter fuertemente espinoso de la superficie lateral hace difícil saber si las espinas más próximas al borde son equivalentes a las presentes en los ejemplares juveniles de Hollinella (Hollinella) y $H$. (Praeholinella).

Dimensiones (en mm)

Adultos con velum incurvado

Media Máx. Mín. Desv.tip. Coef.var

$\begin{array}{lllllrr}\mathrm{L} & 1,28 & 1,31 & 1,19 & 0,041 & 3,206 & 9 \\ \mathrm{H} & 0,793 & 0,81 & 0,78 & 0,016 & 1,993 & 9\end{array}$

Adultos con velum no incurvado

$\begin{array}{ccccccc} & \text { Media } & \text { Máx. } & \text { Mín. } & \text { Desv.tip. Coef.var } & \begin{array}{c}\mathrm{n}^{\circ} \text { ej. } \\ \text { med. }\end{array} \\ \text { L } & 1,18 & 1,22 & 1,09 & 0,051 & 4,321 & 7 \\ \text { H } & 0,737 & 0,78 & 0,69 & 0,038 & 5,178 & 7\end{array}$

Juveniles (varios estadios de crecimiento) Media Máx. Mín. Desv.tip. Coef.var nºj. med. 22

$\begin{array}{lllllll}\mathrm{L} & 0,83 & 1,03 & 0,47 & 0,175 & 21,022 & 22 \\ \mathrm{H} & 0,49 & 0,62 & 0,28 & 0,1 & 20,614 & 22\end{array}$

Ejemplares figurados: 
DPO 32.462 (Holotipo) $\mathrm{L}=1,3 ; \mathrm{H}=079$

DPO 32.467 (Paratipo) $\mathrm{L}=1,13 ; \mathrm{H}=0,69$

DPO 32.475 (Paratipo) $\mathrm{L}=1,02 ; \mathrm{H}=0,6$

DPO 32.473 (Paratipo) $\mathrm{L}=0,90 ; \mathrm{H}=0,52$

\section{Discusión}

Además del contorno general, la lobación y la configuración de las estructuras adventrales, los rasgos más distintivos de la nueva especie son el carácter fuertemente espinoso del domicilium y la ornamentación de las estructuras adventrales de los caparazones adultos con velum fuertemente incurvado.

\section{Subgénero Hollinella (Hollinella) Coryell, 1928 Hollinella (Hollinella) llanisca n. sp.} Fig. 5

Derivatio nominis: El nombre específico deriva de Llanes, en cuyo concejo se encuentra situada la sección de la Playa de la Huelga.

\section{Material}

Holotipo: valva izquierda de ejemplar adulto con velum incurvado en posición distal, DPO 32.482 (Figs. 5A, B). Paratipos: 30 valvas, izquierdas y derechas, de ejemplares adultos, pertenecientes a machos y hembras (DPO 32.483$32.494,32.532,32.533$ ), todos ellos encontrados en la muestra B-260.

Localidad típica: Sección de La Playa de la Huelga, aproximadamente $850 \mathrm{~m}$ al N del pueblo de Hontoria (Fig. 1) (Llanes, Asturias).

Estrato típico: "Calizas del Cuera". Niveles fuertemente silicificados con abundante fauna coralina, en la parte alta de la sección de Hontoria, unos $15 \mathrm{~m}$ por encima de la última intercalación siliciclástica. Moscoviense Superior (tramos equivalentes a la parte alta del horizonte de Podolsk o a la parte baja del horizonte de Myachkovo). Muestra B-260 (Fig. 2).

\section{Diagnosis}

Especie de Hollinella (Hollinella) con L3 muy prominente en sentido lateral, pero no muy extenso longitudinalmente y que casi no sobrepasa el borde dorsal. Superficie preaductorial uniformemente convexa. L1 casi inexistente. No hay separación de L1 y L2 o la separación es muy tenue. Superficie lateral papilosa; papilas dispuestas de un modo bastante regular. Dimorfismo poco marcado. Velum relativamente ancho en los dos dimorfos adultos y aumentando bruscamente de anchura (en sentido radial) en la región anterodorsal. En los individuos de velum ancho, la región anteroventral del velum está algo incurvada en dirección sagital, sin formar estructura cerrada o casi cerrada. El otro dimorfo adulto carece de esta incurvación.

A species of Hollinella (Hollinella) with L3 projecting well above the lateral surface, extending slightly beyond the dorsal border and not very long. Preaductorial area evenly convex. L1 inconspicuous. Lateral surface papillose. Papillae regularly arranged on the lateral surface. Dimorphism not very apparent. Adults with rather wide velum in both sexes, sharply increasing in width in the anterodorsal part. Anterovental part of the velum slightly incurved inwards in one sex and not incurved inwards in the other sex.

\section{Descripción}

Caparazón de tamaño medio. Borde dorsal recto. Bordes libres convexos, casi rectos en posición ventral. Paso del borde ventral a los bordes anterior y posterior gradual. Borde posterior uniformemente convexo. Borde anterior convexo, casi recto en el cuarto anterodorsal. El arco que forma el borde anterior es claramente más largo que el que forma el borde posterior. Ángulos cardinales obtusos, provistos de dos pequeñas espinas que no se conservan en todos los ejemplares y que se dirigen en sentido dorsoanterior y dorsoposterior. L3 grande, destacando fuertemente de la superficie lateral, muy prominente en sentido lateral, pero poco extendido en sentido longitudinal y sin sobrepasar apenas el borde dorsal. Área preaductorial uniformemente convexa, sin diferenciación, o casi sin diferenciación de L1 y L2. L1 casi inexistente. S2 bien marcado. S1 inexistente. Superficie lateral cubierta de numerosas papilas dispuestas de modo bastante uniforme. Borde posterior provisto de espinas marginales bien desarrolladas.

Aunque existe dimorfismo velar, es poco patente y puede pasar desapercibido caso de no disponer de ejemplares con velum bien conservado. El velum comienza en el ángulo anterodorsal y se extiende hasta el límite de los bordes ventral y posterior, donde termina en un fuerte espolón dirigido posteroventralmente. Región dorsoanterior del velum muy estrecha (en sentido radial), aumentando bruscamente de anchura hasta la región ventroanterior, donde alcanza su máximo, y disminuyendo levemente hasta la terminación ventroposterior. El velum está fuertemente alabeado, especialmente en las formas de velum más ancho. En el cuarto anterodorsal, su borde distal es casi perpendicular al plano sagital, en la región ventral es debilmente oblicuo al mismo, dirigido en sentido lateral. El dimorfismo es más acusado en la región anteroventral. En esta región, uno de los dimorfos (formas con velum ancho)

Figura 6. Hollinella (Hollinella) aff. bassleri (Knight). A-C. Vistas lateral externa, interna y vista interna ligeramente inclinada de valva izquierda (ejemplar DPO 32.511). D, E. Vista ventral y detalle de la estriación longitudinal del velum de una valva derecha (ejemplar DPO 32.512). F.G. Vistas dorsales de una valva izquierda y otra derecha (ejemplares DPO 32.514a y 32.514b). H, I. Vista lateral interna y externa de una valva derecha (DPO 32.498). J, K. Vista ventral de dos valvas derechas (DPO 32.501 y DPO 32.500). L. Vista dorsal de valva derecha (DPO 32.503). Todas las figuras son ejemplares adultos. Las figs. A-G corresponden a formas con velum incurvado en dirección sagital, el resto, a formas con velum no incurvado. Todas las figuras aproximadamente $\mathrm{x} 70$, excepto figura $\mathrm{E}$ (aproximadamente x116). 

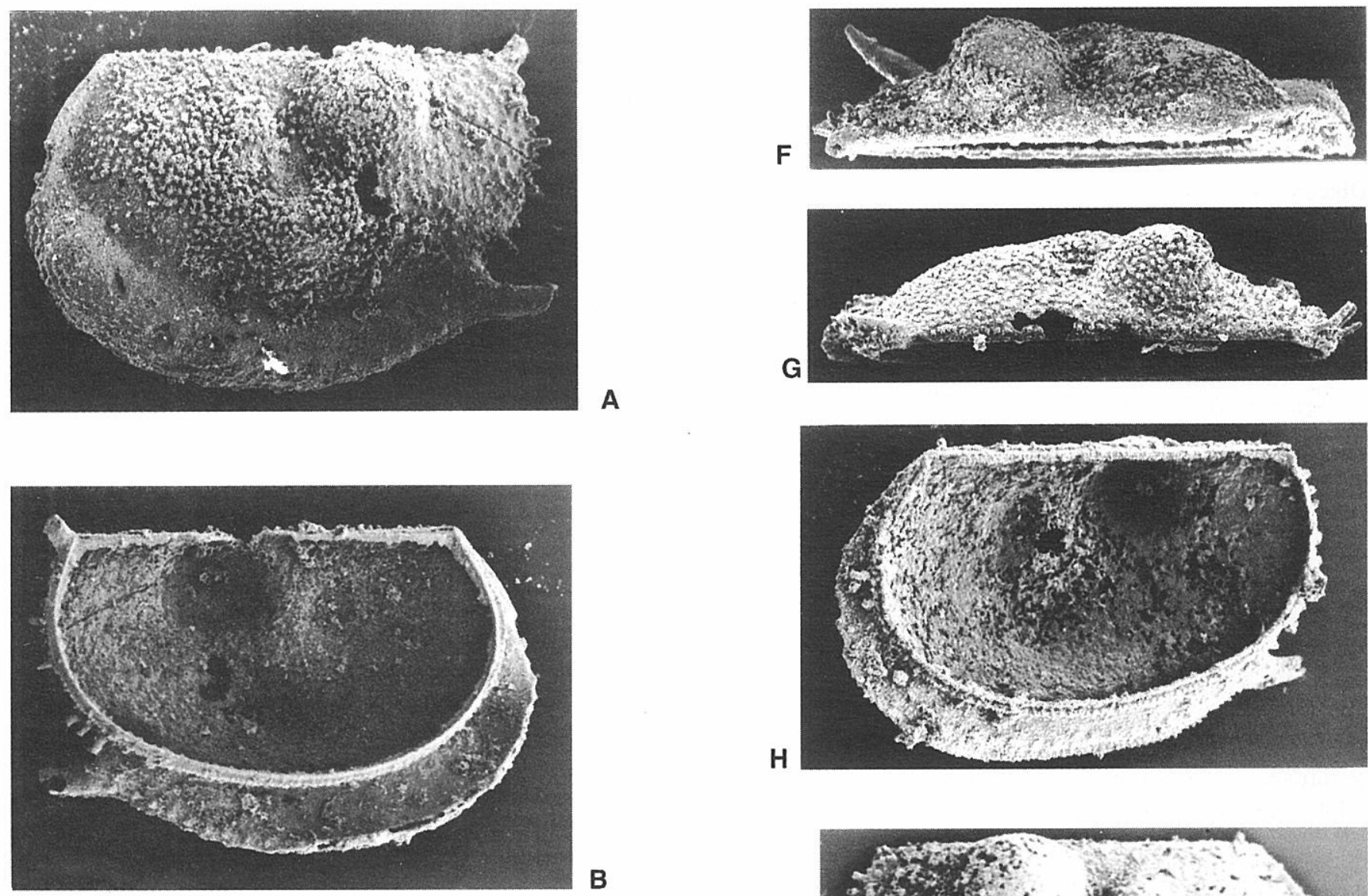

A
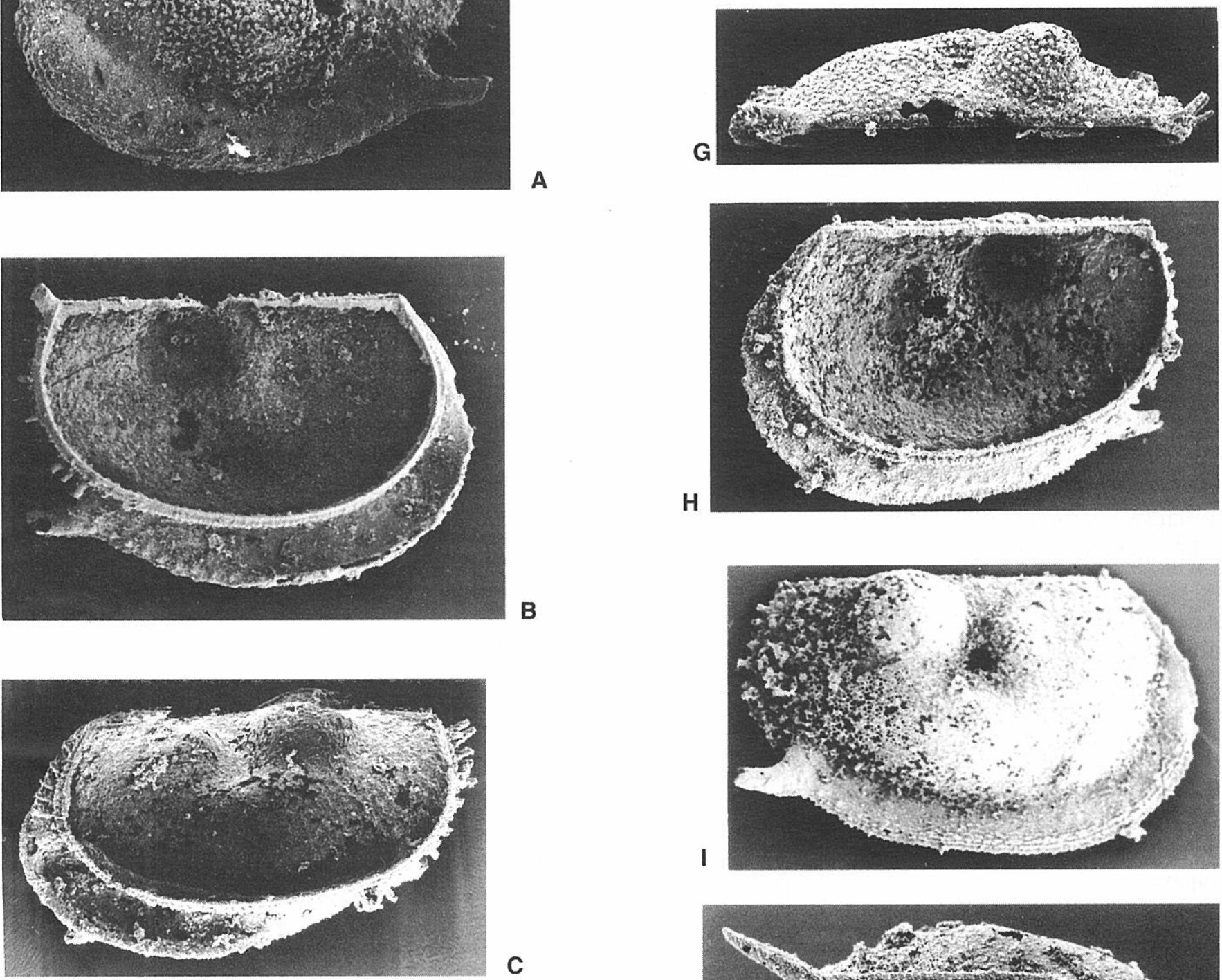

C
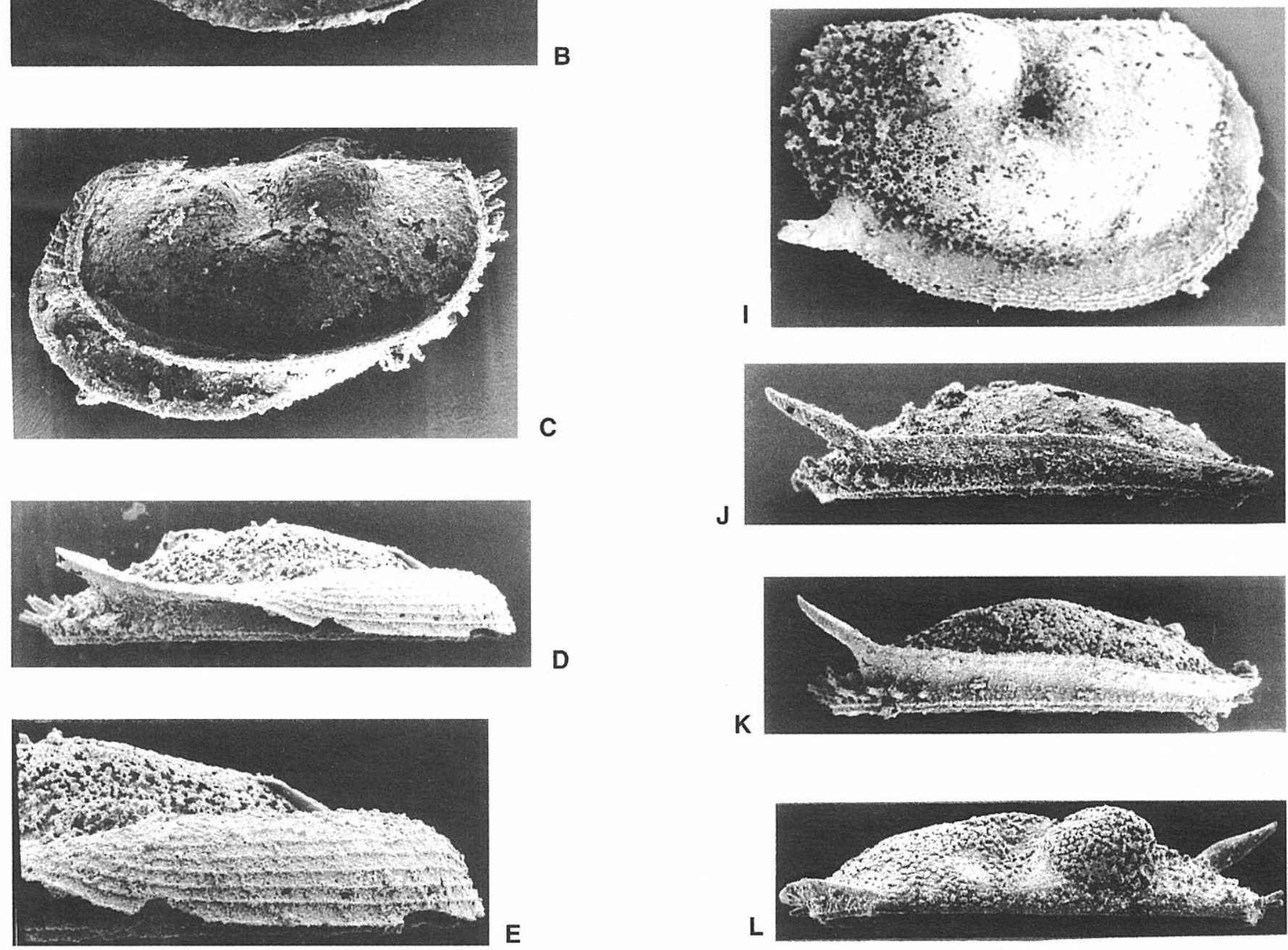

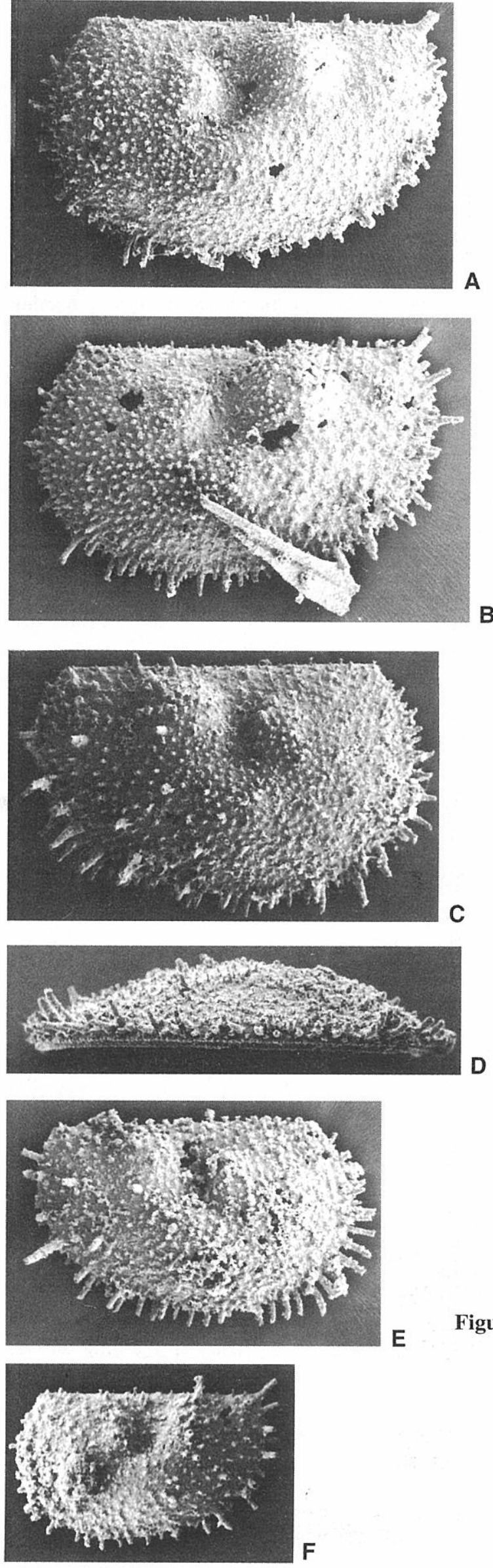

tiene el borde distal del velum débil, pero claramente incurvado en sentido sagital, aunque sin llegar a constituir una cavidad. El otro dimorfo adulto carece de dicha incurvación (o es mucho menos acusada).

Junto con los especímenes adultos de esta especie se encontraron ejemplares juveniles de Hollinella (Hollinella) (Fig. 7), pero no pudieron diferenciarse de los de Hollinella (Hollinella) aff. bassleri, presentes en la misma muestra.

Dimensiones (en mm)

\begin{tabular}{|c|c|c|c|c|c|c|}
\hline & \multicolumn{6}{|c|}{ Adultos de velum ancho } \\
\hline & Media & Máx. & Mín. & Desv.tip. & Coef.var & $\mathrm{n}^{\mathrm{o}}$ ej. \\
\hline $\mathrm{L}$ & 0,91 & 0,96 & 0,81 & 0,048 & 5,232 & 12 \\
\hline $\mathrm{H}$ & 0,6 & 0,62 & 0,56 & 0,027 & 4,438 & 12 \\
\hline & \multicolumn{6}{|c|}{ Adultos de velum estrecho } \\
\hline & Media & Máx. & Mín. & Desv.tip. & Coef.var & $n^{\circ}$ ej. \\
\hline $\mathrm{L}$ & 0,86 & 0,91 & 0,81 & 0,034 & 3,913 & 6 \\
\hline 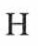 & 0,55 & 0,59 & 0,47 & 0,044 & 7,957 & 6 \\
\hline
\end{tabular}

Ejemplares figurados:

DPO 32.482.(Holotipo) $\mathrm{L}=0,93 ; \mathrm{H}=0,64$

DPO 32.483 (Paratipo) $\mathrm{L}=0,85 ; \mathrm{H}=0,57$

DPO 32.485 (Paratipo) $\mathrm{L}=0,83 ; \mathrm{H}=0,52$

DPO 32.493 (Paratipo) $\mathrm{L}=0,90 ; \mathrm{H}=0,61$

DPO 32.494 (Paratipo) L=0,90; $\mathrm{H}=0,52$

\section{Discusión}

Los ejemplares encontrados presentan las características diagnósticas de Hollinella $(H$.$) ex gr.$ dentata Coryell en el sentido de Bless y Jordan (1972), a saber: ausencia de un L1 marcado y de velum fuertemente incurvado en uno de los dimorfos adultos; igualmente tienen, como es usual en dicho grupo de especies, un domicilium provisto de ornamentación.

El holotipo de Hollinella (H.) dentata es un ejemplar juvenil y, en consecuencia, no muestra las características de las estructuras adventrales. Si bien nuestro material tiene algunas de las características consideradas por Bless y Jordan (1972) como diagnósticas de dicha especie (L2 fundido con L1, domicilium granuloso, estructuras adventrales alabeadas hacia el exterior o débilmente incurvadas en la porción anteroventral del caparazón), tanto en la figuración de Coryell (Coryell, 1928, Lam. 51, fig. 1) como en los ejemplares posteriormente asignados a esta especie es visible un L2 más desarrollado que en el

Figura 7. Ejemplares juveniles de Hollinella (Hollinella). Deben representar juveniles de $H$. (H.) llanisca o $H$. (H.) aff. bassleri, que no pudieron ser diferenciados. Todos ellos fueron encontrados en la muestra B-260. A, B. Vistas laterales de valvas izquierdas probablemente pertenecientes al último estadio juvenil (DPO 32.524 y 32.522 ). C, D. Vistas lateral y ventral de valva derecha perteneciente al mismo estadio ontogenético (DPO 32.521). E, F. Vistas laterales de una valva derecha y otra izquierda pertenecientes a estadios previos (DPO 32.526, 32.523). Todas las figuras aproximadamente $\mathrm{x} 80$. 
material de la sección de la playa de la Huelga. Como hicieron notar Bless y Jordan (op cit.), el domicilium de Hollinella (H.) dentata es muy semejante al de Hollinella $(H$.) bassleri, pero las estructuras adventrales en uno de los dimorfos adultos de la última especie están fuertemente incurvadas, llegando a conectar sus bordes en posición anteroventral.

Los ejemplares descritos tienen también fuertes semejanzas con Hollinella (H.) nowatensis Coryell y Osorio, 1932, en lo que se refiere al contorno lateral, extensión e incurvación de las estructuras adventrales y desarrollo de L2 y L3, pero de acuerdo con Bless y Jordan (1972, p.40) esta última especie tiene un lóbulo ventral prominente.

El material aquí estudiado muestra, así mismo, considerables semejanzas con la forma de los Alpes Cárnicos descrita por Fohrer (en prensa) como Hollinella (H.) aff. cristinae Bless, 1965. Sin embargo, en esta última especie el velum aumenta gradualmente en anchura (en sentido radial) en las proximidades del ángulo cardinal anterior, contra lo que sucede en la especie cantábrica, en la que el velum aumenta bruscamente en la misma región; además, a juzgar por la figura de Fohrer, en el material de los Alpes Cárnicos el velum es más estrecho y parece menos incurvado (es posible no obstante que el ejemplar figurado por dicha autora no conserve la totalidad de las estructuras adventrales).

Hollinella $(H$.$) cristinae Bless tiene L2 más$ desarrollado y el velum parece extenderse más en dirección posterior.

\section{Hollinella (Hollinella) aff. bassleri (Knight, 1928)}

Fig. 6

\section{Material}

1 caparazón adulto con velum incurvado (DPO 32.517, muestra B-260); 2 caparazones adultos con velum no incurvado (DPO 32.496, muestra B-260; DPO 32.505, muestra B-260a), 44 valvas aisladas de ejemplares adultos con velum no incurvado, muestras B-260 y B-260a (DPO $32.495-32.507)$ y 55 valvas adultas con velum incurvado, muestras B-260 y B-260a (DPO 32.508-32.516, DPO $32.518,32.519)$.

\section{Dimensiones (en mm)}

Adultos con velum incurvado

Media Máx. Mín. Desv.tip. Coef.var nºj.

$\begin{array}{llllllr} & & & & & \text { med. } \\ \text { H } & 0,98 & 1,06 & 0,87 & 0,044 & 4,455 & 31 \\ & 0,65 & 0,72 & 0,59 & 0,037 & 5,664 & 31\end{array}$

Adultos con velum no incurvado

Media Máx. Mín. Desv.tip. Coef.var $n^{\circ}$ ej.

$\begin{array}{lllllrr}\text { L } & 0,96 & 1 & 0,87 & 0,029 & 3,042 & 24 \\ \text { H } & 0,6 & 0,62 & 0,56 & 0,027 & 4,438 & 24\end{array}$

Ejemplares figurados:

DPO $32.498 \mathrm{~L}=0,95 ; \mathrm{H}=0,57$

DPO $32.500 \mathrm{~L}=0,92 ; \mathrm{H}=0,52$

DPO $32.501 \mathrm{~L}=0,92 ; \mathrm{H}=0,54$

DPO $32.511 \mathrm{~L}=0,95 ; \mathrm{H}=0,62$

DPO $32.512 \mathrm{~L}=0,95 ; \mathrm{H}=0,62$

\section{Discusión}

Los ejemplares encontrados coinciden, salvo en los rasgos ornamentales, con los descritos por FernándezLópez y Sánchez de Posada (1987) como Hollinella (H.) aff. bassleri, hasta el punto de que creemos que pueden ser coespecíficos. El material estudiado por Fernández López y Sánchez de Posada tiene la superficie lateral lisa, mientras que el de Hontoria está provisto de una marcada ornamentación (tanto en el domicilium como en el velum). La superficie lateral de los ejemplares de Hontoria es granulosa, con gránulos muy prominentes y densos, especialmente en el área situada por delante de L3. Por detrás, suelen parecer pequeñas papilas o espinas, en lugar de gránulos, constituyendo una ornamentación menos densa que la de la región anterior.

Por otra parte, las estructuras adventrales de los especímenes de Hontoria tienen una estriación longitudinal muy marcada y continua (incluso en la porción inflada) que no pudo ser observada en el material del Bashkiriense del $\mathrm{N}$ de León (Fernández López y Sánchez de Posada, 1987). En conjunto, los especímenes de Hontoria están mejor conservados que los que ya eran conocidos de la Zona Cantábrica y no puede descartarse que estas diferencias sean debidas a la conservación. Por ello preferimos designar el material aquí descrito con el mismo nombre. Debe advertirse, en cualquier caso, que la ornamentación del domicilium hace que el material de Hontoria sea muy semejante (o idéntico) a la especie americana.

\section{CONCLUSIONES}

Al menos 3 especies de Hollinella: Hollinella (Truyolsella) truyolsi, Hollinella (H.) llanisca y Hollinella $(H$.$) aff. bassleri, están presentes en la parte$ alta de la sucesión de la Playa de la Huelga.

Hollinella (Truyolsella) truyolsi es la especie tipo de un nuevo subgénero de Hollinella cuyos estadios juveniles tienen gran similitud con los de Hollinella (Keslingella) y Gortanella. La semejanza en las estructuras adventrales de los estadios juveniles de Hollinella (Keslingella) y Hollinella (Truyolsella) nos lleva a suponer que ambos subgéneros se encuentran filogenéticamente relacionados. Probablemente el segundo se originó del primero, conocido ya en el Silúrico Superior.

Hollinella (H.) llanisca parece relacionada con el complejo de Hollinella $(H$.) cristinae y $H$. (H.) dentata, con las que tiene gran parecido.

Hollinella $(H$.$) aff. bassleri presenta fuertes$ afinidades con un grupo de especies de Hollinella ampliamente representado en los EE. UU. de N América 
en materiales de edad comprendida entre el Desmoinesiense y el Pérmico, y apunta a la existencia de conexiones entre las faunas americanas y europeas en el Carbonífero Superior.

Finalmente, Hollinella $(H$.$) aff. bassleri y Hollinella$ (H.) llanisca parecen estrechamente relacionadas con Hollinella (H.).ulrichi y Hollinella (H.) aff. cristinae, halladas en los Alpes Cárnicos, lo que subraya las similitudes entre las faunas cantábricas y cárnicas.

Las tres especies presentes en Hontoria (como las tres presentes en el Gzheliense de los Alpes Cárnicos, descritas por Fohrer (1991 y en prensa) o las existentes en el Pérmico de las Montañas del Bükk, descritas por Kozur, 1985) muestran que los hollinellidos están bien representados en ambientes de plataformas carbonatadas del Paleozoico Superior. Aunque el hecho era sospechado (dado que el género fue capaz de vivir en prácticamente todos los medios marinos), los hallazgos de hollinellidos diversificados en calizas no son frecuentes. Como afirman Bless y Jordan (1972) "It is not impossible that in part selective sampling has led to certain hypothesis about the paleoecological factors controlling the distribution of hollinellid ostracodes. For example, calcified ostracodes cannot be freed from a limestone sample. Hence, data about the distribution of ostracodes in limestones depend entirely on the discovery of silicified or pyritized specimens in these rocks".

\section{AGRADECIMIENTOS}

Deseamos hacer patente nuestro sincero agradecimiento a todas aquellas personas que de un modo $u$ otro han contribuido a la realización de este trabajo. A Beate Föhrer por sus impresiones acerca de las relaciones entre la fauna de ostrácodos de Hontoria y la de los Alpes Cárnicos, a la Editora de la Revista por sus atinadas recomendaciones, a los revisores del trabajo por sus útiles sugerencias, a José Marín por su asistencia en la confección de las figuras, al Servicio de Microscopia electrónica de la Universidad de Oviedo (y, en particular a D. Alfredo Quintana), así como a D. Manuel Alvárez Castañón por su paciente asistencia en el MEB y en el revelado de las fotos, respectivamente.

El presente trabajo se ha realizado dentro de los proyectos PB 941337 de la DGICYT, IR-98515-1 de la Universidad de Oviedo y PICG 421.

\section{BIBLIOGRAFÍA}

Barrois, C. 1882. Recherches sur les terrains anciens des Asturies et de la Galice. Mémoires de la Societé Géologique du Nord, 2, 1, 1-630.

Becker, G. 1978. Flachwasser-Ostracoden aus dem hohen Westfal Asturiens (Kantabrisches Gebirges, N-Spanien). 1. Palaeocopida. Senckenbergiana lethaea, 59 (1/3), $37-$ 69.

Bless, M.J.M. 1965. On two new species of marine ostracodes in the Carboniferous of Asturias. Leidse Geologische Mededelingen, 33, 77-182.
Bless, M.J.M. 1967. On the marine beds of some cyclothems in the Central Carboniferous Basin of Asturias with special reference to their ostracode fauna. Notas $y$ Comunicaciones del Instituto Geológico y Minero de España, 99-100, 91-134.

Bless, M.J.M. 1968. On two hollinid ostracode genera from the Upper Carboniferous of northwestern Spain. Leidse Geologische Mededelingen, 43, 157-212.

Bless, M.J.M. 1969. On a case of dimorphism in the last juvenile stage of a hollinid ostracode from the Namurian B-C of the La Camocha Mine (Gijón, N. Spain). Breviora Geologica Asturica, 13, 31-38.

Bless, M.J.M., and Calver, M. 1970. A Hollinid ostracod from the Middle Coal Measures of Britain. Bulletin of the Geological Survey of Great Britain, 32, 61-67.

Bless, M.J.M. and Jordan, H. 1970. Stratigraphical and taxonomical remarks on the ostracode genus Hollinella Coryell. Mededelingen Rijks Geologische Dienst, N. S., 21, 81-91.

Bless, M.J.M. and Jordan, H. 1972. Ostracodes of the Family Hollinellidae. Mededelingen Rijks Geologische Dienst, Ser. C, 3 (1), 1-155.

Coryell, H.N. 1928. Some new Pennsylvanian Ostracoda. Journal of Paleontology, 2, 377-381.

Coryell, H.N. and Osorio, G.A. 1932. Pennsylvanian ostracoda. An Ostracoda-fauna of the Nowata Shale. American Midland Naturalists, 13, 25-41.

Fernández López, M.G. y Sánchez de Posada, L.C., 1987. Hollinomorpha y Kirkbyocopina (Ostracoda) del Bashkiriense del N. de León (Cordillera Cantábrica, NW de España). Revista Española de Micropaleontología, 19, 465-485.

Fohrer, B. 1991. Verkieselte Flachwasserostracoden und ihre Begleitfauna und -flora aus dem Oberkarbon der Karnischen Alpen (Nassfeld - Region). Abhandlungen der Geologischen Bundesanstalt, 46, 1-107.

Fohrer, B. (en prensa). Ostracoden aus dem Oberkarbon und Unterperm der Karnischen Alpen (Österreich, Italien): Systematik, Biostratigraphie und Paläokologie. Jahrbuch Geologische Bundesastalt.

Julivert, M. 1971. Décollement tectonics in the hercynian Cordillera of northwest Spain. American Journal Science, 270, 1-29.

Kozur, H. 1985. Neue Ostracoden-Arten aus dem Oberen Mittelkarbon (Höheres Moscovian), Mittel- und Oberperm des Bükk-Gebirges. Geologische Paläontologische. Mitteilungen Innsbruck, 2, 1-145.

Lotze, F. 1945. Zur Gliederung der Varisziden der Iberischen Meseta. Geotektektonische Forschungen, 6, 78-92. (Trad. española: Observaciones respecto a la división de las varíscides de la Meseta Ibérica. Publicaciones Extranjeras sobre Geología de España. Consejo Superior de Investigaciones Científicas. Instituto Lucas Mallada de Investigaciones Geológicas, 5, 149-166).

Martínez Chacón, M.L. 1975. Avisyrinx n. gen. (Syringothyrididae, Brachiopoda) del Carbonífero de Asturias (España). Breviora Geologica Asturica, 19, 3340 . 
Martínez Chacón, M.L. 1977. New Carboniferous stenoscismatacean brachiopods from Oviedo and León, Spain. Palaeontology, 20, 209-223.

Martínez Chacón, M.L. 1979. Braquiópodos carboníferos de la Cordillera Cantábrica (Orthida, Strophomenida y Rhynchonellida). Memoria del Instituto Geológico y Minero de España, 96, 1-291.

Martínez Chacón, M.L. 1988. Braquiópodos del Carbonífero Medio del corte de la Playa de La Huelga (Asturias, NW de España). Geogaceta, 5, 13-16.

Martínez Chacón, M.L. 1990. Braquiópodos carboníferos de la costa E de Asturias (España). I: Orthida, Strophomenida, Rhynchonellida y Athyridida. Revista Española de Paleontología, 5, 91-110.

Martínez Chacón, M.L. 1991. Braquiópodos carboníferos de la costa E de Asturias (España). II. Spiriferida y Terebratulida. Revista Española de Paleontología, 6, 5988 .

Navarro, D., Leyva, F. y Villa, E. 1986 Cambios laterales de facies en el Carbonífero del Oriente de Asturias. Trabajos de Geología, Universidad de Oviedo, 16, 87-102.

Pérez Estaún, A., Bastida, F., Alonso, J.L., Marquínez, J., Aller, J., Alvarez Marrón, J., Marcos., A and Pulgar, J.A. 1988. A thin-skinned tectonics model for an arcuate fold and thrust belt: the Cantabrian Zone. Tectonics, 7, 517537.

Rodríguez, S. 1984. Corales Rugosos del Carbonífero del Este de Asturias. Editorial de la Universidad Complutense de Madrid, Tesis Doctoral, 109/84, 1-528.

Rodríguez, S. y Ramírez, C. 1987. Los siringopóridos de la sección de la Playa de la Huelga (Carbonífero, Asturias, Noroeste de España). Boletín de la Real Sociedad Española de Historia Natural, Sección geológica, 83, 57-82.

Ruggieri, G. 1966. Nuovo genere di ostracode del Carbonifero Superiore delle Alpi Carniche. Revista Italiana di Paleontologia, 72, 1-8.

Sánchez de Posada, L.C. 1977. Ostracodes from the Upper Carboniferous of La Camocha Coal Mine (Asturias, N. Spain). Revista Española de Micropaleontología, 9, 411438.

Sánchez de Posada, L.C., Martínez Chacón, M.L., Méndez Fernández, C., Menéndez Alvárez, J.R., Truyols, J. and Villa, E. 1990. Carboniferous pre-Stephanian rocks of the Asturian-Leonese Domain (Cantabrian Zone). In: Pre-Mesozoic Geology of Iberia (Eds. R.D. Dallmeyer and E. Martínez García). Springer Verlag, 24-33.

Sánchez de Posada, L.C., Martínez Chacón, M.L., Méndez Fernández, C., Menéndez Alvárez, J.R., Río, L.M., Truyols, J. y Villa, E. 1996. El Carbonífero marino del ámbito astur-leonés (Zona Cantábrica): síntesis paleontológica. Revista Española de Paleontología, Número Extraordinario, 82-96.

Villa, E. 1985. Foraminíferos de la región oriental de Asturias (Cordillera Cantábrica, $\mathrm{N}$ de España). Compte Rendu X Congrès International de Stratigraphie et de Géologie du Carbonifère, MADRID, 1983, 1, 333-344.

Villa, E. 1995. Fusulináceos carboníferos del Este de Asturias ( $\mathrm{N}$ de España). Biostratigraphie $d u$ Paléozöique, 13, 1-261. 